\title{
New Implementation of Spin-orbit Coupling Calculation on Multi-configuration Electron Correlation Theory
}

\author{
Qianlong Zhou ${ }^{1}$ and Bingbing Suo ${ }^{1}$
}

${ }^{1}$ Northwest University

May 6, 2021

\begin{abstract}
For treating both relativistic effect and electron correlation, the spin-free exact two-component and spin-dependent first-order Douglas-Kroll-Hess (sf-X2C-so-DKH1) Hamiltonian and the state-interaction (SI) method are combined to calculate the spinorbit coupling (SOC) on multi-configuration electron correlation theory. Here, SOC is evaluated via SI among the spin-free states from the complete active space self-consistent field (CASSCF) calculation, and the dynamic electron correlation could be reckoned via the high-level multi-reference electron correlation method. Work equations to evaluate SOC matrix elements over spin-adapted Gelfand states in the framework of the graphic unitary group approach (GUGA) are presented. Benchmark calculations have verified the validity of the present implementation. As a pilot application, the internally contracted MRCI (icMRCI) with the inclusion of SOC calculation produces the reasonable equilibrium bond length and the harmonic vibrational frequency of the ground state of $\mathrm{AuO}$, as well as the transition energy of $\$ \mathrm{X}^{\wedge} 2 \backslash \mathrm{Pi}_{-}\{3 / 2\} \backslash$ leftarrow ${ }^{\wedge} 2 \backslash \mathrm{Pi}_{-}\{1 / 2\} \$$.
\end{abstract}

\section{Hosted file}

soc-guga-bsuo.pdf available at https://authorea.com/users/412190/articles/520957-newimplementation-of-spin-orbit-coupling-calculation-on-multi-configuration-electroncorrelation-theory

\section{Hosted file}

soc-guga-bsuo.tex available at https://authorea.com/users/412190/articles/520957-newimplementation-of-spin-orbit-coupling-calculation-on-multi-configuration-electron-

correlation-theory 